\title{
Diagnóstico da tuberculose em pessoas idosas: barreiras de acesso relacionadas aos serviços de saúde*
}

\author{
TUBERCULOSIS DIAGNOSIS IN THE AGED: BARRIERS TO ACCESSING \\ HEALTH SERVICES
}

\author{
EL DIAGNÓSTICO DE LA TUBERCULOSIS EM LAS PERSONAS MAYORES: LAS \\ BARRERAS RELACIONADAS CON EL ACCESO A LOS SERVICIOS DE SALUD
}

\author{
Annelissa Andrade Virgínio de Oliveira ${ }^{1}$, Lenilde Duarte de Sá ${ }^{2}$, Jordana de Almeida \\ Nogueira ${ }^{3}$, Séfora Luana Evangelista de Andrade ${ }^{4}$, Pedro Fredemir Palha ${ }^{5}$, Tereza \\ Cristina Scatena Villa ${ }^{6}$
}

\begin{abstract}
RESUMO
Este estudo tem como objetivo analisar as barreiras ao diagnóstico da tuberculose em idosos relacionadas aos serviços de saúde no município de João Pessoa-PB. Trata-se de pesquisa qualitativa que envolveu sete pessoas idosas doentes de tuberculose. Entrevistas foram utilizadas para coletar informações. O material empírico foi organizado com o software Atlas.ti versão 6.0 e analisado conforme a técnica de análise de discurso. As barreiras relacionadas ao acesso para confirmação diagnóstica foram: horário de funcionamento das unidades de saúde da família; transferência de responsabilidades; visitas domiciliares sem controle de comunicantes; demora do serviço de saúde em suspeitar da doença e repetidas idas do doente ao serviço de saúde para a obtenção do diagnóstico. Apesar de terem sido identificadas barreiras comuns às encontradas por doentes de tuberculose de um modo geral, em virtude da vulnerabilidade dos idosos, sugerem-se que ações de controle sejam empreendidas pelos serviços de saúde de modo a evitar que a doença torne-se um agravo comum para essa população.
\end{abstract}

\section{DESCRITORES}

Tuberculose

Idoso

Diagnóstico

Acesso aos Serviços de Saúde

Saúde da família

\section{ABSTRACT}

This study was performed with the objective to analyze the barriers to diagnosing tuberculosis in the aged and access to health services in the city of João Pessoa, Paraíba, Brazil. This qualitative study included the participation of seven aged women with tuberculosis. Interviews were used for data collection. The empirical material was organized using Atlas.ti 6.0, and analyzed according to the techniques of discourse analysis. The identified barriers related to the access to health services to confirm the diagnosis were: the operating hours of family health units; transferred responsibilities; home visits without controlling communicants; delay of the health service in suspecting the disease and the patient's repeated visits to the health center before being informed about the diagnosis. Despite the identification of common barriers that tuberculosis patients of all ages must deal with, because of the vulnerability of the elderly, health services should implement control actions so as to prevent the disease becoming a common condition in this population.

DESCRIPTORS
Tuberculosis
Aged
Diagnosis
Health Services Accessibility
Family health

\section{RESUMEN}

Se objetiva analizar las barreras al diagnóstico de tuberculosis en ancianos relacionadas a servicios de salud en João Pessoa-Paraíba. Investigación cualitativa involucrando siete ancianos enfermos de tuberculosis. Informaciones recolectadas mediante entrevistas. El material empírico fue organizado con el software Atlas.ti 6.0 y sometido a técnica de análisis de discurso. Las barreras relacionadas al acceso para confirmación diagnóstica fueron: horario de funcionamiento de las unidades de salud de la familia; transferencia de responsabilidades; visitas domiciliarias sin control de comunicantes; demora del servicio sanitario en sospechar la enfermedad y repetidas visitas del enfermo al servicio de salud para obtener el diagnóstico. A pesar de haber sido identificados obstáculos similares a los encontrados por tuberculosos en general, en virtud de su vulnerabilidad, se sugiere que las acciones de control en ancianos sean emprendidas por los servicios de salud intentando evitar que la enfermedad se convierta en una patología común en dicho segmento poblacional.

DESCRIPTORES
Tuberculosis
Anciano
Diagnóstico
Accesibilidad a los Servicios de Salud
Salud de la familia

*Extraído da dissertação “Diagnóstico da tuberculose em pessoas idosas: barreiras de acesso”, Programa de Pós-Graduação em Enfermagem da Universidade Federal da Paraíba, 2011. ${ }^{1}$ Enfermeira. Mestranda em Enfermagem pelo Programa de Pós-Graduação em Enfermagem da Universidade Federal da Paraíba. Bolsista CAPES. João Pessoa, PB, Brasil. annelissa ufpb@hotmail.com ${ }^{2}$ Enfermeira. Professora Doutora do Departamento de Enfermagem de Saúde Pública e Psiquiatria e do Programa de Pós-Graduação da Universidade Federal da Paraíba. João Pessoa, PB, Brasil. lenilde sa@yahoo.com.br ${ }^{3}$ Enfermeira. Professora Doutora do Departamento de Enfermagem Médico-Cirúrgica e Administração e do Programa de Pós-Graduação da Universidade Federal da Paraíba. João Pessoa, PB, Brasil. jal_nogueira@yahoo.com.br ${ }^{4}$ Graduanda em Enfermagem da Universidade Federal da Paraíba. Bolsista de Iniciação Científica PIBIQ/CNPq. João Pessoa, PB, Brasil. seforaejoab@hotmail.com ${ }^{5}$ Enfermeiro. Professor Doutor do Departamento de Enfermagem Materno Infantil e Saúde Pública e do Programa de Pós-Graduação em Enfermagem da Escola de Enfermagem de Ribeirão Preto da Universidade de São Paulo. Ribeirão Preto, SP, Brasil. palha@eerp. usp.br ${ }^{6}$ Enfermeira. Doutora em Enfermagem em Saúde Pública pela Escola de Enfermagem de Ribeirão Preto da Universidade de São Paulo. Ribeirão Preto, SP, Brasil. tite@eerp.usp.br 


\section{INTRODUÇÃO}

O Brasil é um dos 22 países priorizados pela Organização Mundial de Saúde (OMS) para o controle da tuberculose que apresentam $80 \%$ da carga mundial da doença ${ }^{(1)}$. Em 2007, o Brasil notificou 72.194 casos novos, correspondendo a um coeficiente de incidência de 38/100.000 hab. Estes indicadores colocam o Brasil na 19a posição em relação ao número de casos e na 104 으 posição em relação ao coeficiente de incidência ${ }^{(1)}$.

O Estado da Paraíba (PB), por sua vez, também não apresenta um panorama favorável ao controle da TB. Em 2009, o coeficiente de incidência de TB para todas as formas foi de 27,9/100.000 habitantes e o coeficiente de mortalidade por TB de $2,01^{(2)}$. Além disso, as taxas de cura $(62,88 \%)$ e abandono do tratamento da TB $(12,43 \%)$ nesse Estado contrariam as recomendações da OMS, que preconiza percentual de cura superior a $85 \%$ dos casos detectados e redução da taxa de abandono a menos de $5 \%$ dos $\operatorname{casos}^{(3)}$.

Em 2009, no município de João Pessoa, capital do Estado da Paraíba, o contexto epidemiológico apresentou uma taxa de incidência de TB por todas as formas de 56,39/ 1.000.000 habitantes e taxas de cura $(56 \%)$ e abandono $(8,5)^{(2)}$ também dissonantes do que é preconizado. Esta situação reflete fragilidades nas ações de controle da TB nesse Município, comum à realidade de outros grandes centros urbanos com dificuldades em controlar a doença, principalmente entre os grupos vulneráveis, a exemplo da população de rua, pessoas com HIV e AIDS e idosos.

Seguindo a tendência mundial de enveIhecimento da população, a incidência de TB no Brasil começa a se deslocar para pessoas idosas. No Brasil, no ano de 2007, o número total de casos de tuberculose (TB) foi de 72.140, sendo que 7.862 (9\%) ocorreram em pessoas com idade igual ou superior a 60 anos, fato que revela a importância epidemiológica da doença entre pessoas idosas no País ${ }^{(4)}$. Nesse sentido, a questão do envelhecimento vem sendo articulada ao aumento do número de casos de TB e aos casos de retardo do diagnóstico. Salienta-se que o diagnóstico da TB é sabidamente mais difícil nessa faixa etária, fato que determina elevada mortalidade e internações ${ }^{(5)}$.

O retardo no diagnóstico da TB é um dos problemas que acentua a gravidade da doença. Entre os aspectos relacionados ao evento - retardo no diagnóstico da TB - destacam-se aqueles inerentes ao sistema de saúde, como: dificuldade de acesso; acolhimento inadequado do doente; baixa prioridade na procura de sintomáticos respiratórios (SR) e de contatos intra-domiciliares; baixo nível de suspeição diagnóstica de TB, correspondendo ao aumento do período entre a primeira visita ao serviço de saúde e o início do tratamento antituberculose ${ }^{(6)}$.
Estudos avaliativos realizados por integrantes do Grupo de Estudos e Qualificação em Tuberculose da Paraíba - Grupo TB/PB apontam problemas que concorrem para a falta de acesso do doente ao serviço de saúde, destacando-se fragilidades de acessibilidade geográfica, econômica e organizacional ${ }^{(7-8)}$.

Assim, frente ao aumento do número de casos de TB em idosos e à dificuldade do diagnóstico nessa faixa etária, este estudo tem como objetivo analisar as barreiras ao diagnóstico da TB em pessoas idosas relacionadas aos serviços de saúde no município de João Pessoa-PB.

\section{MÉTODO}

Este estudo foi conduzido pela pesquisa qualitativa no campo analítico do discurso (AD) de matriz francesa $a^{(9)}$. No processo de análise do material empírico utilizou-se a técnica de $A D^{(9)}$, indicada em pesquisas qualitativas pelas possibilidades de relacionamento dos materiais que envolvem valores, juízos necessários e preferíveis como argumentos ou como meios capazes de revelar a visão de mundo e, portanto, a posição ideológica do sujeito discursivo.

Elegeu-se como cenário do estudo o município de João Pessoa - PB, considerado prioritário pelo Ministério da Saúde (MS) para o controle da TB. Atualmente, nesse Município, as ações de controle da tuberculose são realizadas pelas equipes de Saúde da Família (SF), Centros Especializados, hospitais de referência e laboratórios que realizam a baciloscopia.

Participaram do estudo sete pessoas idosas doentes de TB. Foram considerados os seguintes critérios para a inclusão dos sujeitos: ser maior de 60 anos; estar em ou ter concluído o tratamento de TB em unidades de saúde da família do município de João Pessoa e ser domiciliado nesse Município. Para identificação dos sujeitos, contou-se com o apoio da coordenadora de Seção Tuberculose do referido Município, que buscou junto ao sistema de informação de agravos de notificação (SINAN) informações sobre doentes que atendiam aos critérios de inclusão do estudo. Foram identificados 10 idosos. Destes, um havia falecido, um se mudou e não foi possível o contato um doente, resultando assim, em sete sujeitos que participaram da pesquisa, identificados pela letra $C$ e dispostos na sequência em que foram entrevistados de $\mathrm{C} 1 \mathrm{a}$ C7.

Considerando os aspectos éticos e em conformidade com a Resolução 196/96 CNS, o projeto de pesquisa foi submetido ao Comitê de Ética do Centro de Ciências da Saúde da Universidade Federal da Paraíba - CCS/ UFPB, sendo aprovado em 17 de dezembro de 2008, sob protocolo $n^{\circ} 0589$.

A coleta de informações foi realizada nos meses de dezembro de 2010 a fevereiro de 2011 , tendo sido a en-
Diagnóstico da tuberculose em pessoas idosas: barreiras de acesso relacionadas aos serviços de saúde Oliveira AAV, Sá LD, Nogueira JA, Andrade SLE, Palha PF, Villa TCS 
trevista semidirigida o recurso empregado para esse fim. Seguindo os preceitos éticos, as entrevistas só foram iniciadas após os sujeitos concordarem em participar do estudo e assinarem o termo de consentimento livre e esclarecido (TCLE). As entrevistas foram realizadas por duas pesquisadoras, uma estudante de mestrado e uma bolsista de iniciação científica, no local de residência dos sujeitos e gravadas individualmente, com uso de aparelho audioportátil e MP4.

Para a análise das informações inicialmente foram realizadas leituras exaustivas do material empírico resultante da transcrição dos depoimentos dos sujeitos, com a finalidade de identificar sinais, vestígios e pistas. Realizado este processo, foi constituído o corpus discursivo. A partir do corpus discursivo foram observados os enunciados em relação às barreiras ao acesso relacionadas aos serviços de saúde.

$\mathrm{Na}$ codificação do corpus discursivo foi empregado o software Atlas.ti versão 6.0., indicado para sistematização das informações em pesquisas qualitativas. Com o uso dessa ferramenta foram criados sete códigos: Horário de funcionamento das USF e turno de trabalho do usuário; Transferência de responsabilidades; Sinal/Sintoma determinante para a busca do serviço de saúde; A não realização de visitas domiciliares; A realização de visitas domiciliares sem controle de comunicantes; A demora do serviço de saúde relacionada ao atraso na suspeita da doença e repetidas idas serviço de saúde para a obtenção do diagnóstico. Após a codificação das sete entrevistas, foram gerados relatórios, sobre os quais foi aplicada a técnica de análise de discurso.

Na segunda etapa da análise procurou-se observar nos discursos os processos parafrásicos, polissêmicos e metafóricos, os quais possibilitaram a identificação da seguinte formação discursiva: elementos relacionados aos serviços de saúde no acesso ao diagnóstico por parte das pessoas idosas com TB e suas implicações no retardo do diagnóstico. Em seguida os enunciados que caracterizavam os discursos dos sujeitos foram analisados quanto à posição ideológica do sujeito, contradições, silenciamento, memória discursiva e opacidade do discurso.

\section{RESULTADOS}

Os fragmentos discursivos a seguir mostram elementos relacionados aos serviços de saúde no acesso ao diagnóstico por parte das pessoas idosas e implicados no retardo do diagnóstico da TB.

Quadro 1 - Códigos e fragmentos discursivos das pessoas idosas quanto às barreiras no acesso ao diagnóstico relacionadas ao serviço de saúde - João Pessoa, 2011

\begin{tabular}{|c|c|}
\hline Códigos & Fragmentos Discursivos das Pessoas Idosas \\
\hline $\begin{array}{l}\text { Horário de funcionamento das USF e } \\
\text { turno de trabalho do usuário }\end{array}$ & Eu estava trabalhando, quando eles vinham aqui de manhã. C6 \\
\hline Transferência de responsabilidades & 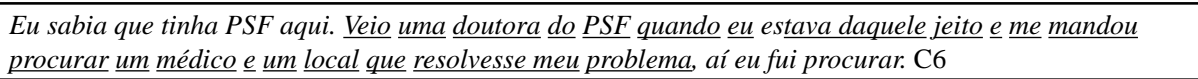 \\
\hline $\begin{array}{l}\text { Sinal/Sintoma determinante para a } \\
\text { busca do serviço de saúde }\end{array}$ & 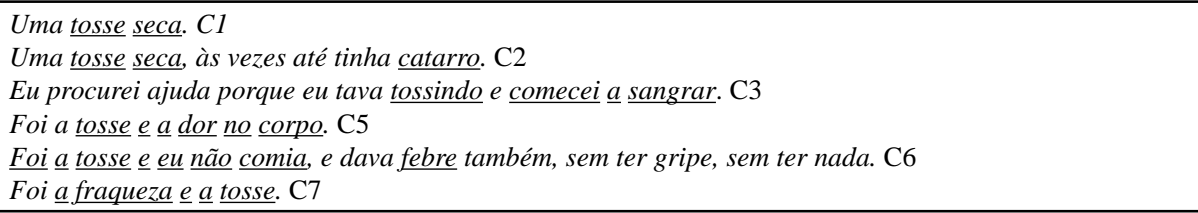 \\
\hline Não realização de visitas domiciliares & 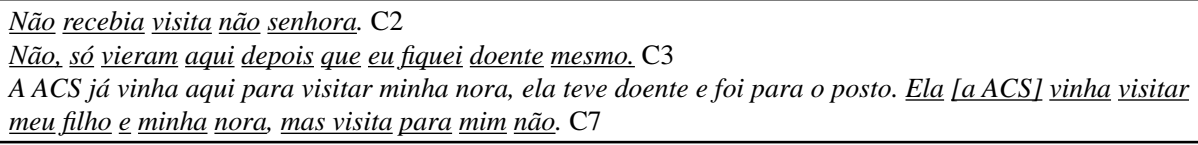 \\
\hline $\begin{array}{l}\text { Visitas domiciliares sem controle de } \\
\text { comunicantes }\end{array}$ & $\begin{array}{l}\text { Não [...] sim, recebia visita da ACS porque minha filha se tratava lá [de TB] e ela vinha. Também da } \\
\text { enfermeira. C1 } \\
\text { A ACS sempre vinha quando meu filho tava doente }[\mathrm{de} \text { TB] e sempre vem ver meu neto que tem necessi- } \\
\text { dades especiais e minha filha. C6 }\end{array}$ \\
\hline $\begin{array}{l}\text { Demora do serviço de saúde relacionada } \\
\text { ao atraso na suspeita da doença }\end{array}$ & 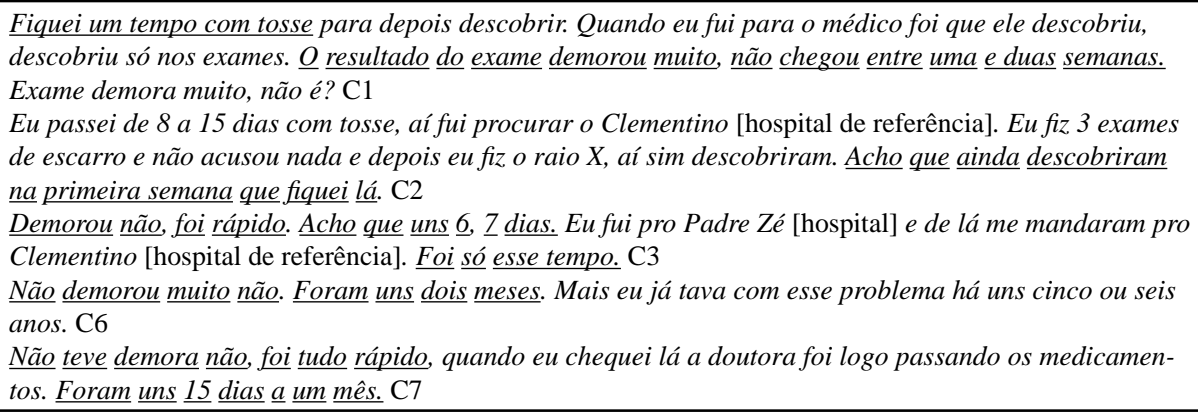 \\
\hline $\begin{array}{l}\text { Repetidas idas serviço de saúde para a } \\
\text { obtenção do diagnóstico }\end{array}$ & 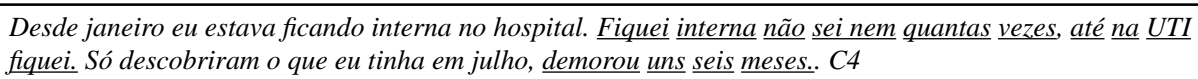 \\
\hline
\end{tabular}




\section{DISCUSSÃO}

Elementos relacionados aos serviços de saúde no acesso ao diagnóstico por parte das pessoas idosas com TB e suas implicações no retardo do diagnóstico.

A primeira barreira de acesso aos serviços de saúde para o diagnóstico da TB citada no fragmento discursivo do colaborador 6 trata da dificuldade quanto ao horário de funcionamento das USF, revelando fragilidade nas dimensões organizacional e de disponibilidade de acesso aos serviços de saúde. A disponibilidade em relação à conveniência de horário, ou seja, dias ou horários em que o serviço de saúde está aberto para prestar atendimento, pode configurar um aspecto desfavorável e limitante ao diagnóstico. Nesse sentido, chama atenção o fato de que nem sempre o horário de trabalho das equipes e de funcionamento da unidade de saúde consegue atender às necessidades da população(10).

A questão da limitação no horário de funcionamento das USF também foi identificada como uma dificuldade constrangedora do efetivo acesso aos serviços da atenção básica $(A B)$ em estudo realizado em São Paulo(11). $O$ fato do colaborador 6 enunciar que as visitas a sua residência ocorriam sempre pela manhã - horário em que estava no trabalho - sinaliza problemas relacionados à organização interna dos processos de trabalho das equipes, bem como revela que a conjuntura atual de inserção diferenciada dos idosos no mercado de trabalho e a participação dessas pessoas em atividades voltadas à terceira idade precisam ser considerada pelos serviços de saúde.

A origem deste conflito entre oferta, demanda e necessidades expõe a estrutura rígida que em geral conforma o atuar das unidades de saúde e parece impedir os profissionais de cuidar das pessoas considerando sua singularidade e suas necessidades mais concretas.

Estudo realizado em cinco municípios brasileiros mostrou que a descentralização das ações de TB para os serviços da atenção básica parece não apresentar desempenho satisfatório para o acesso ao diagnóstico, não sendo a forma de organização da atenção à TB fator determinante para garantia de acesso ao diagnóstico precoce ${ }^{(6)}$. Uma vez que a descentralização dos cuidados aos doentes de TB para as USF deu-se na tentativa de aproximar o doente do serviço e ampliar as condições de acesso, o fato do horário de funcionamento ser um fator limitante representa uma contradição dentro desse modelo de assistência. Assim, evidencia-se que a resolução desse problema precisa ser compartilhada com os usuários, a fim de que suas reais necessidades sejam levadas em consideração(11).

Outro elemento identificado nos discursos que se relaciona aos serviços de saúde no acesso ao diagnóstico por parte das pessoas idosas com TB - e tem implicações no retardo do diagnóstico - é a transferência de responsabilidade. No fragmento discursivo do colaborador 6, a sequência discursiva de referência leva a interpretar que o profissional de saúde não se coloca na posição de diagnosticar a TB, transferindo a responsabilidade para o usuário em buscar um serviço que lhe garanta o diagnóstico.

O diagnóstico da TB é de responsabilidade dos profissionais das USF que, em caso de suspeita clínica, devem coletar amostra de escarro para exame já no primeiro contato com o usuário, o que termina por corroborar para um diagnóstico precoce ${ }^{(3)}$. Entretanto, na opacidade desse discurso, percebe-se a não responsabilização dos profissionais da ESF com as ações de diagnóstico da TB, o que implica falta de qualificação profissional.

Apesar das ações de diagnóstico e controle da TB terem sido descentralizadas para as USF, não é possível que as mesmas sejam viabilizadas sem disposição e efetiva participação dos profissionais envolvidos. É preciso que os profissionais que atuam nos serviços da atenção básica sejam qualificados para exercer seu papel, pois a falta de conhecimento por parte destes relaciona-se a falhas na detecção precoce dos sintomáticos respiratórios bem como na definição do diagnóstico.

A qualificação dos profissionais é um aspecto que interfere na incorporação de ações de controle da TB na Atenção Básica e o conhecimento insuficiente dos profissionais de saúde sobre os fatores de risco da TB e o processo do diagnóstico. aliado à reduzida prescrição do exame baciloscópico, concorrem para retardar o diagnóstico $^{(12)}$ e o início do tratamento.

Nesse sentido, a qualificação profissional desenvolvida por meio da educação permanente é um importante requisito no contexto da Atenção Básica, pois promove autonomia, habilidade técnica e interpessoal, criatividade, qualidade e a humanização necessária para que as equipes de saúde tenham condições de planejar e gerenciar a assistência aos doentes de TB na área em que atuam ${ }^{(13)}$. Todavia, é imprescindível que as especificidades da TB no idoso, bem como em outros grupos vulneráveis, façam parte do escopo das ações de educação permanente para esses profissionais.

Outro elemento importante identificado nos discursos é a inadequada avaliação dos casos sintomáticos respiratórios na comunidade. Observa-se nos fragmentos discursivos que a tosse associada a algum outro sinal/sintoma - principalmente a presença de sangue e fraqueza - foi o principal motivo que levou o idoso a procurar o serviço de saúde. O fato revela que a busca dos idosos sintomáticos respiratórios ocorre de forma passiva e demonstra a presença marcante de um modelo assistencial baseado na demanda espontânea, que se afirma na espera que o doente de TB procure pelo serviço de saúde ao perceber algum sinal ou sintoma da doença. Ressalta-se que a busca passiva é a investigação de TB em indivíduos sintomáticos respiratórios que procuraram o serviço de saúde devido a tosse. Já a busca ativa é a busca de TB pulmonar em indivíduos sintomáticos respiratórios que não procuraram o serviço de saúde ${ }^{(14)}$.
Diagnóstico da tuberculose em pessoas idosas: barreiras de acesso relacionadas aos serviços de saúde Oliveira AAV, Sá LD, Nogueira JA, Andrade SLE, Palha PF, Villa TCS 
De acordo com as recomendações da III Diretrizes para Tuberculose da Sociedade Brasileira de Pneumologia e Tisiologia ${ }^{(14)}$, o atraso na identificação de casos de TB pulmonar ocorre devido à avaliação inadequada dos casos sintomáticos respiratórios ou à procura tardia do serviço de saúde, sendo a busca ativa uma atividade multiprofissional com o objetivo de diagnosticar a TB precocemente, especialmente nos grupos com maior risco de adoecimento, como é o caso dos idosos.

A detecção de casos entre sintomáticos respiratórios e contatos está prevista nas visitas domiciliares dos Agentes Comunitários de Saúde $(\mathrm{ACS})^{(3)}$, entretanto, os fragmentos discursivos dos colaboradores C2, C3 e C7 evidenciam a não realização das visitas com foco também no idoso, o que contraria não só as diretrizes do programa de controle da TB pelos serviços de Atenção Básica, mas a Política Nacional de Saúde do Idoso que recomenda que o cuidado a essa parcela da população seja feito de modo integral ${ }^{(15)}$. Assim, evidencia-se que o profissional de saúde não valoriza o idoso como grupo vulnerável à TB, sendo imprescindível, portanto, que o planejamento das ações de controle da TB aponte para a diversidade, incluindo diferentes etnias, população de rua, pessoas privadas de liberdade e os diferentes grupos etários.

Com o intuito de detectar mais precocemente os casos de TB, os membros das equipes de saúde devem incorporar à rotina dos serviços de saúde a busca ativa de sintomáticos respiratórios e seus contatos ${ }^{(3)}$, ou seja, toda pessoa, parente ou não, que coabita com um doente de $\mathrm{TB}^{(3)}$. É assustador constatar a realização de visitas domiciliares sem o rastreio dos contatos, como é expresso nos fragmentos discursivos dos colaboradores C1 e C6.

Embora a saúde do idoso seja área estratégica da Atenção Básica de Saúde, verifica-se, nas unidades discursivas, o apagamento de ações voltadas ao controle de comunicantes, no caso, pessoas idosas que convivem com familiares com TB.

Uma vez que um caso de TB é confirmado, deve-se proceder à investigação epidemiológica, incluindo o exame das pessoas que convivem com o doente. Essa investigação objetiva identificar as possíveis fontes de infecção devendo ser feita entre todos os contatos de todo o caso novo de TB e, prioritariamente, para os que convivem com doentes bacilíferos, especialmente os contatos intra-domiciliares, por apresentarem maior probabilidade de infecção e adoecimento( ${ }^{(3)}$.

Segundo o Ministério da Saúde do Brasil, a investigação do contato é uma das estratégias mais apropriadas de vigilância para interromper a transmissão e o desenvolvimento subsequente da TB, só perdendo em efetividade para a busca ativa de sintomáticos respiratórios ${ }^{(3)}$. 0 exame de contato definido como atividade de vigilância epidemiológica pelo Programa Nacional de Controle da Tuberculose (PNCT) tem seus procedimentos normatizados em publicações oficiais do Ministério da Saúde. Contudo, esse exame não tem sido priorizado pelos serviços de saúde da atenção básica.
Uma vez que os idosos que participaram do estudo fazem parte de um dos grupos etários considerados mais vulneráveis à TB, é preciso refletir sobre o cuidado prestado a esses contatos, principalmente no que concerne à vigilância da saúde, pois é sabido que as organizações de serviços de saúde enfrentam alguns desafios operacionais. Há falta de sistematização das atividades de avaliação dos contatos em termos de monitoramento e ações de vigilância epidemiológica ${ }^{(16)}$.

Os fragmentos discursivos deixam claro que os profissionais não investem na busca casos de TB, o que contraria a concepção de Vigilância da Saúde, uma vez que realizar busca ativa de sintomáticos respiratórios deve ser uma atitude permanente e incorporada à rotina de atividades de todos os membros das equipes de saúde ${ }^{(3)}$. Nota-se, portanto, uma dificuldade na incorporação da busca de sintomáticos respiratórios pelas equipes, que pode estar associada à simplificação desta ação, uma vez que a busca parece uma atividade simples, mas na prática caracteriza-se como uma atividade complexa que requer conhecimentos que vão além da execução de procedimentos técnicos.

A busca de sintomáticos respiratórios exige uma abordagem à família que transcende o saber biológico. Caracteriza-se como uma sequência de atividades que vão desde o contato no domićlio, a orientação sobre a doença, sinais e sintomas, modo de transmissão, identificação dos sintomáticos respiratórios, orientação sobre a coleta de escarro, encaminhamento e recebimento do material pela USF, fluxo do exame ao laboratório de análise, recebimento do resultado pela unidade e usuário, até ao encaminhamento para o seguimento ambulatorial dos casos diagnosticados ${ }^{(3)}$.

Com o intuito de detectar mais precocemente os casos de TB, os membros das equipes de saúde devem incorporar a busca de casos novos de TB à rotina dos serviços de saúde. A incorporação da busca ativa de sintomáticos respiratórios no controle da TB no âmbito da ESF requer, fundamentalmente, que seja assumido um conjunto de ações, tanto no âmbito externo (abordagem no domicilio junto às famílias, identificação do suspeito) como no âmbito interno dos serviços de saúde (recebimento de material, encaminhamento ao laboratório, diagnóstico) para que seja garantida a longitudinalidade da atenção e a formação de vínculo ${ }^{(17)}$. Ressalta-se ainda a importância de uma vigilância epidemiológica mais efetiva, que inclua visitas domiciliares rotineiras ${ }^{(16)}$, sobretudo em moradias da área de abrangência da Unidade de Saúde da Família com pessoas idosas.

Os discursos dos idosos quanto ao tempo que levou para que obtivessem o diagnóstico revelam uma variação de uma semana até seis meses e, mais que quantificar o tempo, os fragmentos discursivos dos colaboradores $\mathrm{C} 1$, C2, C3, C6 e C7 revelam que para alguns deles esse tempo não parece longo. Percebe-se no fio dos discursos que o atraso aqui relatado ocorreu por parte do serviço de saúde. Salienta-se ainda que esse bloco discursivo, além de reforçar a posição resignada do doente em relação ao 
tempo do diagnóstico, revela o retardo do diagnóstico da TB em idosos também no âmbito hospitalar.

A demora do sistema de saúde é entendida como o intervalo de tempo entre a primeira consulta em qualquer unidade de saúde até a data do diagnóstico, sendo que intervalos superiores a 3 dias são considerados indicativos de um diagnóstico tardio(18). Partindo dessa definição é alarmante a constatação de que todos os idosos em estudo tiveram o atraso em seu diagnóstico. A opacidade do discurso sugere que, além dos profissionais de saúde não estarem atentos à identificação de sintomáticos respiratórios idosos, familiares, cuidadores e demais contatos, não citados, provavelmente desconhecem sinais e sintomas sugestivos da doença. Tal fato concorre para que familiares e cuidadores de pessoas idosas possam receber qualificação de modo a abreviar a confirmação diagnóstica da doença.

O atraso no diagnóstico é um reflexo claro dos pontos fracos no sistema de saúde ${ }^{(18)}$. Estudos que analisaram a demora do serviço de saúde encontraram valores entre 02 a 60 dias, sendo o intervalo de tempo maior para os idosos do que para os não-idosos ${ }^{(18-19)}$. Essa demora, do ponto de vista do sistema de saúde, é atribuível ao atraso na suspeita da doença ${ }^{(19)}$. No entanto, mais que refletir os pontos fracos do sistema de saúde, a demora na detecção de casos de TB pode ser considerada um índice da capacidade de diagnosticar a doença e iniciar o tratamento e isso deve ser sistematicamente avaliado principalmente por suas consequências negativas, quais sejam: o agravamento do quadro clínico do doente, maior mortalidade e disseminação sustentada na comunidade, pois pessoas não tratadas que continuam a transmitir a infecção para outras pessoas ${ }^{(19)}$.

Além disso, o fato dos idosos referirem que o diagnóstico não demorou - mesmo que a tardança observada varie de uma semana a dois meses - constitui uma contradição e mostra que são passivos em aceitar as decisões dos serviços de saúde. Evidencia-se que há um silenciamento dos idosos frente à situação, uma vez que é esperado que os usuários ajam como sujeitos ativos e protagonistas das ações de saúde ${ }^{(20)}$. Assim, salienta-se a importância de ações voltadas à transformação do usuário passivo que silencia como partícipe no cuidado a sua saúde. Nesse caso, as equipes de saúde devem fomentar a participação da comunidade nas ações de promoção da saúde.

Verifica-se ainda que os profissionais de saúde não atuam visando à promoção da autonomia do doente, bem como não levam em consideração suas singularidades na busca pela integralidade do cuidado. É imprescindível, pois, que os profissionais que cuidam do idoso com TB levem em conta suas singularidades, o que permitirá o manejo eficaz da saúde e o estabelecimento de um vínculo efetivo entre os profissionais e os doentes ${ }^{(21)}$.

Ainda em se tratando da demora por parte do serviço de saúde, no fragmento do discurso do colaborador 4 observa-se a necessidade de ir várias vezes ao serviço de saúde para a obtenção do diagnóstico. Destaca-se desse fragmento discursivo a necessidade do usuário ir repetidas vezes ao serviço de saúde em busca de seu diagnóstico, o que acaba por retardar sua confirmação.

$\mathrm{Na}$ opacidade do discurso do colaborador 4 percebe-se ainda o descontentamento com o serviço de saúde. Quando o doente procura o serviço de saúde e tem suas necessidades atendidas, este estabelece uma relação de confiança com os profissionais e com o serviço. Entretanto, se o doente procura o serviço, mas não recebe respostas satisfatórias às suas demandas, cria-se a descrença ${ }^{(22)}$, que pode comprometer a procura pelo serviço de saúde. Assim, fica evidente a necessidade desses serviços se reestruturarem na tentativa de disponibilizar um cuidado integral e resolutivo aos doentes de TB, neste caso, a pessoa idosa que porta a doença e que é extremamente vulnerável ao retardo do diagnóstico da doença, face às barreiras de acesso discutidas nesse estudo.

\section{CONCLUSÃO}

Os fragmentos discursivos analisados permitiram a identificação de barreiras ao acesso relacionadas aos serviços de saúde, quais sejam: dificuldades quanto ao horário de funcionamento das USF; transferência de responsabilidades; inadequada avaliação dos casos sintomáticos respiratórios na comunidade; a não realização de visitas domiciliares; a realização de visitas domiciliares sem controle de comunicantes; a demora do serviço de saúde relacionada ao atraso na suspeita da doença e a necessidade de ir várias vezes ao serviço de saúde para a obtenção do diagnóstico.

Frente às barreiras identificadas, evidencia-se a necessidade de reorganização dos serviços de saúde em âmbito local visto que estes têm privilegiado a atenção à demanda espontânea em detrimento da realização de ações de promoção da saúde, tais como visitas domiciliares e busca ativa de sintomáticos respiratórios. Assim sendo, é necessário que o processo de trabalho das equipes de saúde da família seja revisto, com maior valorização da incorporação dessas ações. Para tanto, ressalta-se a necessidade de que esses profissionais sejam capacitados para realizarem uma efetiva busca ativa de sintomáticos respiratórios com vistas a diagnosticá-los precocemente, evitando o retardo do diagnóstico. Destaca-se ainda que a falta de qualificação dos profissionais implicou em menor suspeita clínica e corroborou para o retardo do diagnóstico, o que reforça a importância do oferecimento de qualificações em TB para os profissionais.

Assim, sugere-se a promoção de qualificações profissionais tendo a TB em idosos como tema e que sejam adequadas à realidade na qual as equipes de Saúde da Família estão inseridas e a reorganização dos processos de trabaIho das equipes, com a incorporação à rotina de visitas domiciliares e busca ativa de sintomáticos respiratórios. 
1. World Health Organization (WHO). Global tuberculosis control: surveillance, planning, financing: WHO report. Geneva: WHO; 2009.

2. Paraíba. Secretaria de Estado da Saúde. Sistema de Informação de Agravos e Notificação (SINAN-Net). Indicadores que caracterizam a situação da tuberculose na Paraíba. João Pessoa; 2011.

3. Brasil. Ministério da Saúde; Secretaria de Políticas de Saúde, Departamento de Atenção Básica. Manual técnico para o controle da tuberculose: cadernos de atenção básica. 6a ed. Brasília; 2002.

4. Codenotti SB. Incidência da tuberculose em idosos. In: Brasil. Ministério da Saúde. Programa Nacional de Controle da Tuberculose (PNCT). Brasília; 2010.

5. Shin JY, Jung SY, Lee JE, Park JW, Yoo SJ, Park HS, et al. Characteristics of pulmonary Webb-Yates M. Tuberculosis in the elderly: a different disease? tuberculosis in elderly people. Tuberc Respir Dis. 2010;69(3):163-70.

6. Pesut DP, Gledović ZB, Grgurević AD, Nagorni-Obradović LM, Adzić TN. Tuberculosis incidence in elderly in Serbia: key trends in socioeconomic transition. Croat Med J. 2008;49(6):807-12.

7. Cantalice Filho JP, Sant'Anna CC, Bóia MN. Aspectos clínicos da tuberculose pulmonar em idosos atendidos em hospital universitário do Rio de Janeiro, RJ, Brasil. J Bras Pneumol. 2007;33(6):699-706.

8. Scatena LM, Villa TCS, Ruffino Netto A, Kritski AL, Figueiredo TMRM, Vendramini SHF, et al. Dificuldades de acesso a serviços de saúde para diagnóstico de tuberculose em municípios do Brasil. Rev Saúde Pública. 2009;43(3):389-97.

9. Figueiredo TMRM, Villa TCS, Scatena LM, Gonzales RIC, Ruffino-Netto A, Nogueira JA, et al. Desempenho da atenção básica no controle da tuberculose. Rev Saúde Pública. 2009; 43(5):825-31.

10. Sá LD, Andrade MN, Nogueira JÁ, Villa TCS, Figueiredo TMRM, Queiroga RPF et al. Implantação da estratégia DOTS no controle da Tuberculose na Paraíba: entre o compromisso político e o envolvimento das equipes do programa saúde da família (1999-2004). Cienc Saude Coletiva. 2011; 16(9):3917-24.
11. Orlandi EP. Análise de discurso: princípios e procedimentos. 8a ed. Campinas: Pontes; 2009.

12. Elias PE, Ferreira CW, Alves MCG, Cohn A, Kishima V, Escrivão Junior $A$, et al. Atenção Básica em Saúde: comparação entre PSF e UBS por estrato de exclusão social no município de São Paulo. Cienc Saude Coletiva. 2006;11(3):633-41.

13. Giatti L, Barreto SM. Saúde, trabalho e envelhecimento no Brasil. Cad Saúde Pública. 2003;19(3):759-71.

14. Monroe AA, Gonzales RIC, Palha PF, Sassaki CM, Ruffino Netto A, Vendramini SHF, et al . Envolvimento de equipes da atenção básica à saúde no controle da tuberculose. Rev Esc Enferm USP. 2008;42(2):262-7.

15. Cotta RMM, Schott M, Azeredo CM, Franceschini SCC, Priore $\mathrm{SE}$, Dias G. Organização do trabalho e perfil dos profissionais do Programa Saúde da Família: um desafio na reestruturação da atenção básica em saúde. Epidemiol Serv Saúde. 2006;15(3):7-18.

16. Conde MB, Melo FAF, Marques AMC, Cardoso NC, Pinheiro VGF, Dalcin PTR, et al. III Diretrizes para Tuberculose da Sociedade Brasileira de Pneumologia e Tisiologia. J Bras Pneumol. 2009;35(10):1018-48.

17. Brasil. Ministério da Saúde. Portaria n. 2.528, de 19 de outubro de 2006. Aprova a Política Nacional de Saúde da Pessoa [Internet]. Brasília; 2006 [citado 2010 ago. 10]. Disponível em: http://www.ufrgs.br/3idade/portaria1395gm.html

18. Mfinanga SG, Mutayoba BK, Kahwa A, Kimaro G, Mtandu R, Ngadaya $E$, et al. The magnitude and factors associated with delays in management of smear positive tuberculosis in Dar es Salaam, Tanzânia. BMC Health Serv Res. 2008;8:158.

19. Gómez AMN, Megías AJ, Soler JC, Augé MC, Fuentes JMA, Galvao MLS, et al. Estudio del retraso diagnóstico de la tuberculosis pulmonar sintomática. Arch Bronconeumol. 2003;39(4):146-52.

20. Ayres JRCM. O cuidado, os modos de ser (do) humano e as práticas de saúde. Saude Soc. 2004;13(3):16-29.

\section{Agradecimentos}

Ao projeto multicêntrico Retardo no diagnóstico da tuberculose: análise das causas em diferentes regiões do Brasil, aprovado e financiado pelo Conselho Nacional de Desenvolvimento Científico e Tecnológico (Edital MCT/CNPq/CT-Saúde/MS/SCTIE/DECIT n. 034/2008).

À Coordenação de Aperfeiçoamento de Pessoal de Nível Superior (CAPES) pelo apoio financeiro. 